\title{
Block Abstraction Memoization for CPAchecker (Competition Contribution)
}

\author{
Daniel Wonisch \\ University of Paderborn, Germany \\ dwonisch@mail.upb.de
}

\begin{abstract}
Block Abstraction Memoization (ABM) is a technique in software model checking that exploits the modularity of programs during verification by caching. To this end, ABM records the results of block analyses and reuses them if possible when revisiting the same block again. In this paper we present an implementation of ABM into the predicate-analysis component of the software-verification framework CPACHECKER. With our participation at the Competition on Software Verification we aim at providing evidence that ABM can not only substantially increase the efficiency of predicate analysis but also enables verification of a wider range of programs.
\end{abstract}

\section{Verification Approach}

Currently, software model checking is getting more and more successful and is getting applied to industrial-size programs. Yet, scalability of the applied methods is still an issue. One approach to improve the scalability of model checking is block abstraction memoization (ABM). ABM exploits the modularity of programs by caching intermediate analyses of blocks. That is, ABM records the results of block analyses as for example analyses of loops or functions, and reuses them if possible when revisiting the same block again. We have implemented ABM into the predicate-analysis component of the software verificationframework CPACHECKER [3, including support for lazy refinements. It shows that $\mathrm{ABM}$ does not only increase the efficiency of the predicate analysis but also allows to successfully analyze programs that were not possible to analyze without.

As illustrative example, consider the C program (fragment) shown in the left of Figure 1 The program consists of three nested while loops, each incrementing a respective counter variable twice. After the execution of all loops it is asserted that the counting variables of the loops are indeed 2. On the right, Figure 1 shows a representation of this program as control-flow automaton (CFA). When analyzing the program using predicate abstraction we can prove the safety of the program with, e.g., the set of predicates $\{i=0, i=1, i=2, j=0, j=$ $1, j=2, k=0, k=1, k=2\}$. While doing so, CPACHECKER will visit, e.g.,

\footnotetext{
* This work was partially supported by the German Research Foundation (DFG) within the Collaborative Research Centre "On-The-Fly Computing" (SFB 901). 

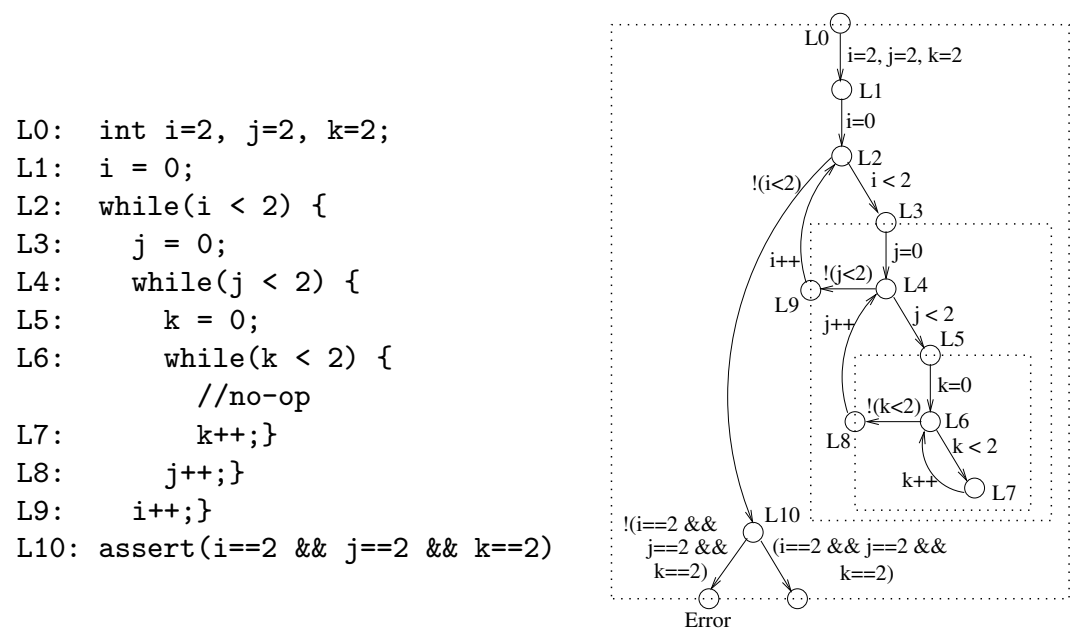

Fig. 1. Program NESTED and its control-flow automaton with 3 blocks

location L5 four times. At each visit the respective predicate abstractions only differ from each other in the valuation of the outer loop counter variables $i$ and $j$. $\mathrm{ABM}$ allows to avoid this redundancy by considering the loop bodies as separate blocks as indicated in the right of Figure 1 by dotted rectangles. ABM initiates at each visit of a block a separate analysis whose result is cached. For example, if $L 3$ is reached with an abstract state $i=0 \wedge j=2 \wedge k=2$, ABM first recognizes that $i$ is irrelevant for the block and thus analyzes the loop body starting with the reduced initial element $j=2 \wedge k=2$. After reaching the end of the block, the final abstract element $j=2 \wedge k=2$ is expanded to the full state space again, i.e., to $i=0 \wedge j=2 \wedge k=2$ ( $i$ cannot change due an execution of this block), and the analysis of the program continues. In the next loop iteration, when reaching $L 3$ again, the cached analysis can be reused and a re-analysis avoided. Similarly, with ABM, the most inner block starting with location L5 has to be analyzed only once. Hence, in this example, the amount of explored abstract elements for ABM-based model checking only grows linearly with the number of nested loops, leading to an exponential speed-up compared to the exponentially growing amount with classical model checking.

\section{Implementation}

We implemented our approach in the program-analysis tool CPACHECKER 3 . CPACHECKER is a framework for configurable program analyses (CPAs) [2]. CPAs allow users to specify different verification approaches in a uniform formalism. ABM is implemented as CPA in CPAchecker in order to benefit from existing verification components. Because ABM always functions as extension CPA (e.g., predicate analysis), it is technically implemented as a WrapperCPA. The wrapped CPA needs to comply with the $C P A W i t h A B M$ interface that basically requires the CPA to provide a reduce and expand operation for its abstract 
elements. So far, we have only implemented this interface in the PredicateAnalysis CPA. However, in principle, we could also easily enable ABM for other analyses (like e.g. shape analysis) by just implementing a reduce and expand operation for abstract elements of the respective domain.

In its supplied configuration, ABM considers loops and functions as blocks. Furthermore, it will consider all those predicate as relevant for a block for which a contained variable occurs in the block. As wrapped CPA we use predicate analysis with adjustable-block encoding (ABE) [1] configured to compute an abstraction at the start and end of a loop or function body. MathSAT 4 (http://mathsat4.disi.unitn.it) is used as underlying SMT solver. Using this configuration, CPACHECKER with ABM performs very well on the ControlFlowInteger and reasonably well on the System C and DeviceDrivers64 benchmark sets. With an incomplete analysis of pointer-aliases and heap structures, our approach is naturally rather unsuccessful for the DeviceDrivers and HeapManipulation sets. Concurrency (Pthreads) is currently not supported at all. Compared to CPACHECKER without ABM, our technique is especially beneficial on the DeviceDrivers64 benchmark set.

\section{Installation Instructions}

ABM is fully integrated into the official source code of CPACHECKER. It can thus be downloaded from the official CPACHECKER webpage http://cpachecker.sosy-lab.org (Apache 2.0 license; Software Systems Lab, University of Passau). We use Revision 4573 for the competition. CPACHECKER can be compiled by executing ant in the checkout folder. To run CPACHECKER with the ABM configuration on a test file, execute scripts/cpa.sh -sv-comp12-abm -heap 12500m source_file in the checkout folder. For the DeviceDrivers64 set, -setprop cpa.predicate. machineModel=64-Linux needs to be specified additionally. Contrary to this, for the DeviceDrivers set the script should be called with the arguments -setprop cpa.predicate.handlePointerAliasing=true, -setprop cpa.abm.blockHeuristic=LoopPartitioning, -sv-comp12-abm-funpoint, and -heap 12500m. Counterexamples, if found, are written to test/output/ErrorPath.txt.

Acknowledgement. We would like to thank Philipp Wendler for his extensive help with the integration of ABM into CPACHECKER.

\section{References}

1. Beyer, D., Keremoglu, M.E., Wendler, P.: Predicate Abstraction with AdjustableBlock Encoding. In: FMCAD 2010, pp. 189-197 (2010)

2. Beyer, D., Henzinger, T.A., Théoduloz, G.: Configurable Software Verification: Concretizing the Convergence of Model Checking and Program Analysis. In: Damm, W., Hermanns, H. (eds.) CAV 2007. LNCS, vol. 4590, pp. 504-518. Springer, Heidelberg (2007)

3. Beyer, D., Keremoglu, M.E.: CPAchecker: A Tool for Configurable Software Verification. In: Gopalakrishnan, G., Qadeer, S. (eds.) CAV 2011. LNCS, vol. 6806, pp. 184-190. Springer, Heidelberg (2011) 